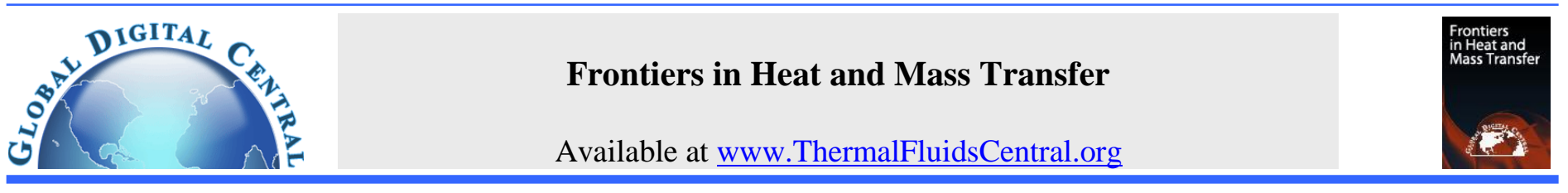

\title{
AN OPTIMIZED TREE-TYPE CYLINDRICAL-SHAPED NANOPOROUS FILTERING MEMBRANE
}

\author{
Yongbin Zhang* \\ College of Mechanical Engineering, Changzhou University, Changzhou, Jiangsu Province, China
}

\section{ABSTRACT}

A tree-type cylindrical-shaped nanoporous filtering membrane is optimized with two levels of branches and a lot of branch pores. In this membrane, the branch pores are parallel with their trunk pore and their radius $R_{b, 1}$ is determined by the filtration requirement; The radius of the trunk pore is optimized according to the radius of its branch pore for achieving the lowest flow resistance of the membrane. The calculations were made respectively for the optimum ratios of the radius of the trunk pore to the radius of the branch pore and for the corresponding lowest flow resistances of the membrane for different operational parameter values. It was found that for given operating conditions, the increase of the number $N$ of the branch pores significantly increases the optimum ratio of the radius of the trunk pore to the radius of the branch pore but significantly reduces the corresponding lowest flow resistance of the membrane; However, an over large $N$ is not beneficial owing to not obviously dropping the flow resistance of the membrane; The choice of the number $N$ of the branch pores may heavily depend on the liquid-pore wall interaction. It was also found that for any given $N$, this membrane has a good capability of liquid-liquid separation when $R_{b, 1}$ is below $2 \mathrm{~nm}$, since its flow resistance for the liquid with a strong interaction with the pore wall can be more than 100 or even more than 1000 times that for the liquid with a weak interaction with the pore wall.
\end{abstract}

Keywords: Membrane; Filtration; Separation; Nanopore; Optimization

\section{INTRODUCTION}

In recent years, nanoporous filtering membranes were in fast development because of their capability of ultimate filtration and their important applications in super purification of water and seawater, hemofiltration, drug delivery and DNA analysis (Adiga et al., 2009; Baker and Bird, 2008; Biffinger et al., 2007; Fissel et al., 2009; Jackson and Hillmyer, 2010). The development was mainly on the membrane fabrication, however the analysis on the characteristics of and the design methods for these membranes were little seen. The shortcomings of nanoporous filtering membranes are their relatively large flow resistance because of the nanometer scale filtration pore radius and thus their comparatively small flux and also their relatively low mechanical strength because of small membrane thicknesses. For overcoming these shortcomings, several improved nanoporous filtering membranes were proposed by using the material of graphene, taking the shape of the filtration pore as conical or using a mixed membrane composed of nanopores and micropores (Li et al., 2004; Surwade et al., 2015; Yang et al., 2006).

In an earlier research (Zhang, 2018a), the author proposed a cylindrical-shaped nanoporous filtering membrane composed of two kinds of pores i.e. the filtration pore and the flow resistance-reducing pore. There is an optimum ratio between the radii of these two pores for achieving the lowest flow resistance of the membrane. This membrane was suggested to have an overall good performance including the improved flux and mechanical strength.

In a recent study (Zhang, 2018b), a tree-type cylindrical-shaped nanoporous filtering membrane was proposed for liquid-particle or liquid-liquid separations. The pores across the thickness of this membrane have the tree structure, and they are divided into one trunk pore and four branch pores. The branch pore is for filtration with the radius on the nanometer scale and with the axial length i.e. pore depth as small as possible. The trunk pore is for collecting the flow out of its branch pores and for reducing the flow resistance of the membrane with considerably larger radius. There is a small angle $\theta$ between the axis of the trunk pore and the axis of its branch pore. This membrane was shown to also have improved flux and mechanical strength.

As a further study, the present paper presents an optimized tree-type cylindrical-shaped nanoporous filtering membrane for liquid-particle separation or for liquid-liquid separation. The geometrical structure of this membrane is similar with that of the membrane proposed by Zhang(2018b). In the present membrane, the branch pores are parallel with its trunk pore, they are for filtration with the radius on the nanometer scale determined by the size of the filtered particle or by the requirement of the liquid-liquid separation, and their number in each pore tree can be relatively big for generating sufficiently high flux of the membrane; The radius of the trunk pore is optimized for achieving the lowest flow resistance of the membrane. This membrane should have substantially improved flux and satisfactory mechanical strength. An analysis is presented for this membrane regarding the determination of the optimum ratio of the radius of the trunk pore to the radius of its branch pore. The calculation was made for the corresponding lowest flow resistances of this membrane for different operational parameter values when the radius of the trunk pore is optimum. Important conclusions are drawn regarding the performance of this membrane.

\section{MEMBRANE PICTURES}

Figure 1 shows an exemplary tree-type cylindrical-shaped nanoporous filtering membrane studied in the present paper. Across the thickness of the membrane are manufactured tree-structure cylindrical pores including one trunk pore and $N$ branch pores (In Fig.1, $N=4$ ); Within the 
membrane, these pores are uniformly distributed. The branch pores are parallel with its trunk pore, they are for filtration with the radius $R_{b, 1}$ on the nanometer scale, their length i.e. pore depth $l_{1}$ should be as small as possible determined by the requirement of the strength of the filtration pore, and their number $N$ can be widely varied depending on the flux requirement. The radius of the trunk pore is $R_{b, 2}$, which is optimized for achieving the lowest flow resistance of the membrane; The depth of the trunk pore is $l_{2}$ The thickness of the membrane is $\left(l_{1}+l_{2}\right)$, which is determined according to the requirement of the mechanical strength of the membrane.

In Fig.1(b), $\Delta_{1}$ and $R_{d}$ are respectively the circumferential distance between the neighboring branch pores and the radius of the distribution circle of the branch pores in each pore tree. As an example, for $N=4, \Delta_{1}$ and $R_{d}$ are determined as follows: $k_{s}=\Delta_{1} / R_{b, 1}$ and $R_{d} / R_{b, 1}=\left(4+2 k_{s}\right) / \pi$; Here, the value of $k_{s}$ is determined according to the requirement of the manufacturing of the branch pores, and it can be between 0.05 and 0.5 for dense branch pore distributions.

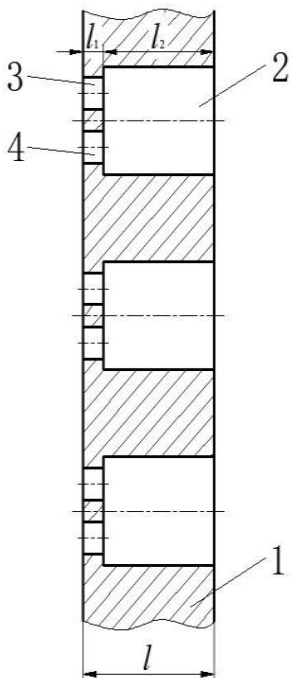

(a) Front view

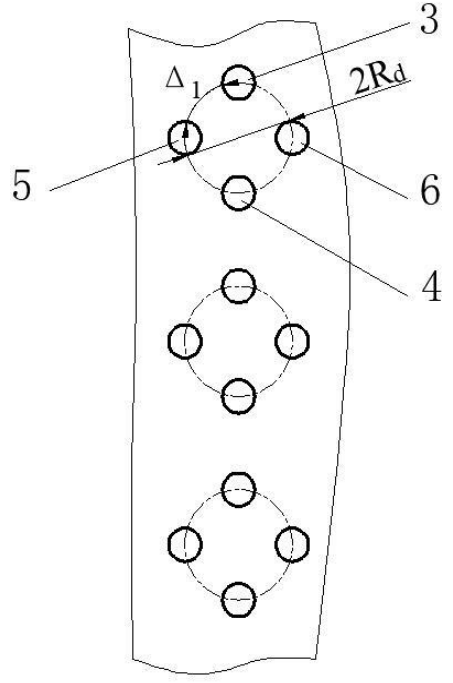

(b) Left-side view
1-Membrane substrate, 2-Trunk pore, 3,4,5,6-branch pores

Fig. 1 An exemplary tree-type cylindrical-shaped nanoporous filtering membrane studied in the present paper.

\section{OPTIMIZATION ANALYSIS}

In the study by Zhang (2018b), a tree-type cylindrical-shaped nanoporous filtering membrane was proposed as Fig. 2(a) shows. In that membrane, there is an angle $\theta$ between the axis of the trunk pore and the axis of its branch pore. According to the analysis made by Zhang(2018b), the flow resistance of the membrane in Fig.2(a) is reduced with the reduction of $\theta$ and $\theta=0^{\circ}$ gives the lowest flow resistance of the membrane for given operational parameter values. For $\theta=0^{\circ}$, the membrane in Fig.2(a) becomes the membrane as shown in Fig.1(a).

The flows inside the branch and trunk pores in the present membrane are normally non-continuum because of the pore radii on the nanometer scale. In principle, the Navier-Stokes equation fails for such flows. In these flows, the liquid across the pore radius becomes noncontinuum and the effect of the liquid-pore wall interaction should be taken into account. The flow equation for a nanoscale flow has been developed (Zhang, 2016); This equation accounts for the effects of the liquid discontinuity and inhomogeneity across the pore radius i.e. the liquid non-continuum effect due to the confinement of the pore wall; It also incorporates the effects of the liquid-pore wall interfacial slippage and the liquid dynamics. When the pore radius is so large that the liquid inside the pore becomes continuum, this equation reduces to the NavierStokes equation. This equation has been validated by molecular dynamics simulations. According to the principle of the transportation in nanotube tree (Zhang, 2017a), which is based on the non-continuum flow described by the developed flow equation (Zhang, 2016), the branch pores with the number $\mathrm{N}$ in each pore tree in Fig.1(a) is equivalent to the straight cylindrical pore with the radius $R_{e q}$ and with the pore depth $l_{1}$. Here, for simplicity, the liquid-pore wall interfacial slippage is neglected, and $R_{e q}$ is thus solved from the following equation (Zhang, 2017a):

$$
\frac{N \cdot C q\left(\bar{R}_{b, 1}\right)\left|S\left(\bar{R}_{b, 1}\right)\right| \bar{R}_{b, 1}^{4}}{C y\left(\bar{R}_{b, 1}\right)}-\frac{C q\left(\bar{R}_{e q}\right)\left|S\left(\bar{R}_{e q}\right)\right| \bar{R}_{e q}^{4}}{C y\left(\bar{R}_{e q}\right)}=0
$$

where $\bar{R}_{b, 1}=R_{b, 1} / R_{c r}, \bar{R}_{e q}=R_{e q} / R_{c r}, R_{c r}$ is the critical radius of the pore for the filtered liquid to become continuum across the pore radius, $C y(\bar{R})=\eta_{b f}^{\text {eff }}(\bar{R}) / \eta, \quad C q(\bar{R})=\rho_{b f}^{\text {eff }}(\bar{R}) / \rho, \quad \rho_{b f}^{\text {eff }} \quad$ and $\eta_{b f}^{\text {eff }}$ are respectively the average density and the effective viscosity of the filtered liquid across the pore radius, $\mathrm{S}$ is the parameter describing the noncontinuum effect of the filtered liquid across the pore radius $(-1 \leq S<0)$, and $\rho$ and $\eta$ are respectively the bulk density and the bulk viscosity of the filtered liquid at the environmental temperature and pressure. According to the developed nanoscale flow equation(Zhang, 2016), the radius $R_{b, 1}$ of the branch pore in Eq.(1) not only can be as small as on the nanometer scale but also can be much larger as on the micrometer or even millimeter scales.

Only when $\bar{R}_{e q} \geq 1, R_{e q}$ can be analytically solved from Eq.(1) (Zhang, 2017a). Otherwise, $R_{e q}$ should be numerically solved from Eq.(1). Here, $R_{e q}$ is generally numerically solved. Thus, the membrane in Fig.1(a) is equivalent to the membrane shown in Fig.2(b) in which the radius of the smaller pore is $R_{e q}$ and the radius of the larger pore is $R_{b, 2}$.

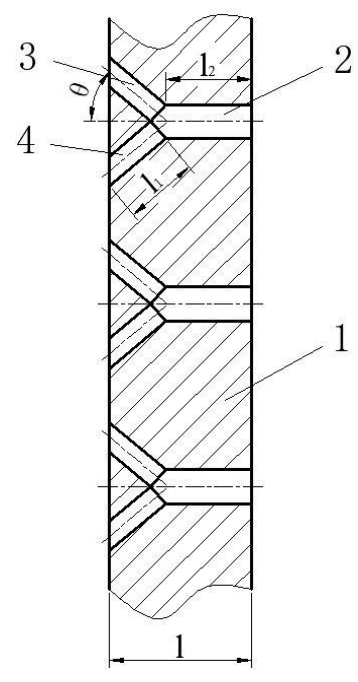

(a)

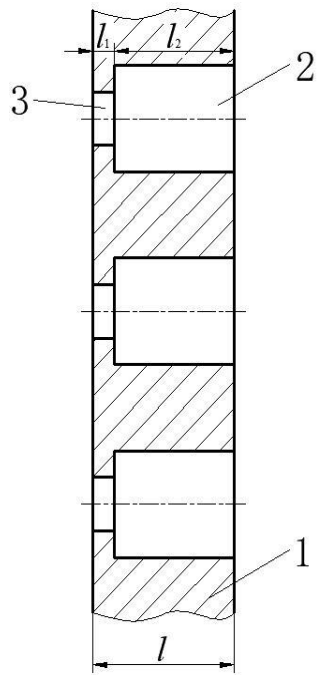

(b)
1-Membrane substrate, 2-Flow resistance-reducing pore, 3,4-Filtration pore

Fig. 2 The ever studied membranes. (a) The tree-type cylindrical-shaped nanoporous filtering membrane (Zhang, 2018b); (b) The cylindricalshaped nanoporous filtering membrane composed of two kinds of pores i.e. the filtration pore and the flow resistance-reducing pore studied by Zhang(2018a).

According to the analysis developed by Zhang(2018a), if the flow resistance of the membrane in Fig.2(b) is defined as $i_{f}=\Delta p / q_{m}$, where $\Delta p$ is the pressure drop on this membrane and $q_{m}$ is the mass flow rate through this membrane, $i_{f}$ is:

$i_{f}=\frac{4 \eta l}{\rho \chi A_{m} R_{r}^{2}} F\left(\frac{R_{b, 2}}{R_{e q}}\right)\left(\frac{\bar{R}_{r}}{\bar{R}_{e q}}\right)^{2}$ 
where $R_{r}$ is a constant reference radius, $\bar{R}_{r}=R_{r} / R_{c r}, A_{m}$ is the area of the membrane surface, $\chi$ is the pore production rate of the membrane surface, and the function $\mathrm{F}$ is:

$$
F\left(\frac{R_{b, 2}}{R_{e q}}\right)=\frac{\lambda_{0} C y\left(\bar{R}_{e q}\right)}{C q\left(\bar{R}_{e q}\right)\left|S\left(\bar{R}_{e q}\right)\right|}\left(\frac{R_{b, 2}}{R_{e q}}\right)^{2}+\frac{\left(1-\lambda_{0}\right) C y\left(\bar{R}_{b, 2}\right)}{C q\left(\bar{R}_{b, 2}\right)\left|S\left(\bar{R}_{b, 2}\right)\right|\left(\frac{R_{b, 2}}{R_{e q}}\right)^{2}}
$$

where $\lambda_{0}=l_{1} / l$ and $\bar{R}_{b, 2}=R_{b, 2} / R_{c r}$.

According to Eq.(3), there is an optimum value of the ratio $R_{b, 2} / R_{e q}$ which gives the minimum value of the function $\mathrm{F}$ and then the lowest flow resistance of the membrane according to Eq.(2).

When $R_{b, 2} \geq 0.9 R_{c r} R_{b, 2} \geq 0.9 R_{c r}, \operatorname{Cy}\left(\bar{R}_{b, 2}\right) \approx 1, \operatorname{Cq}\left(\bar{R}_{b, 2}\right) \approx 1$ and $\left|S\left(\bar{R}_{b, 2}\right)\right| \approx 1$, thus according to Eq.(3), the optimum value of the ratio $R_{b, 2} / R_{e q}$ for yielding the minimum value of the function $\mathrm{F}$ is (Zhang, 2018a):

$$
\left(\frac{R_{b, 2}}{R_{e q}}\right)_{o p t}= \begin{cases}{\left[\frac{\left(1-\lambda_{0}\right) C q\left(\bar{R}_{e q}\right)\left|S\left(\bar{R}_{e q}\right)\right|}{\lambda_{0} C y\left(\bar{R}_{e q}\right)}\right]^{\frac{1}{4}}} & , \text { for } R_{b, 2} \geq R_{e q} \\ 1 \quad & \text { for } R_{b, 2}<R_{e q}\end{cases}
$$

The resulting minimum value of the function $F$ is (Zhang, 2018a):

$$
F_{\min }= \begin{cases}2 \sqrt{\frac{\lambda_{0}\left(1-\lambda_{0}\right) C y\left(\bar{R}_{e q}\right)}{C q\left(\bar{R}_{e q}\right)\left|S\left(\bar{R}_{e q}\right)\right|}} & \text { for } R_{b, 2} \geq R_{e q} \\ \frac{C y\left(\bar{R}_{e q}\right)}{C q\left(\bar{R}_{e q}\right)\left|S\left(\bar{R}_{e q}\right)\right|} & \text { for } R_{b, 2}<R_{e q}\end{cases}
$$

If the dimensionless flow resistance of the membrane is defined as $I_{f}=\rho \chi A_{m} R_{r}^{2} i_{f} /(4 \eta l)$, for the optimum value of $R_{b, 2} / R_{e q}$ shown in Eq.(4), the resulting lowest dimensionless flow resistance of the membrane in Fig.2(b) i.e. the membrane in Fig.1(a) is: $I_{f, \min }=$ $\left(\bar{R}_{r} / \bar{R}_{e q}\right)^{2} F_{\text {min }}$. For yielding the lowest flow resistance of the membrane in Fig.1(a), the optimum ratio of $R_{b, 2}$ to $R_{b, 1}$ is: $\left(R_{b, 2} / R_{b, 1}\right)_{\text {opt }}=$ $\left(R_{e q} / R_{b, 1}\right)\left(R_{b, 2} / R_{e q}\right)_{o p t}$.

\section{CALCULATION}

Exemplary calculations of the values of $\left(R_{b, 2} / R_{b, 1}\right)_{\text {opt }}$ and the corresponding lowest dimensionless flow resistance $I_{f, \min }$ of the membrane in Fig.1(a) have been calculated respectively from Eqs.(4) and (5) for $R_{r}=10 \mathrm{~nm}, \bar{R}_{b, 1}=0.3$, different $\lambda_{0}$ values and different liquid-pore wall interactions when $N$ varies from 1 to 20 . In all these calculations, it was examined that the calculation accuracy is good (Zhang, 2018a).

In the calculations, for whichever liquid-pore wall interaction, $C q(\bar{R})$ is generally expressed as (Zhang, 2018a and 2018b):

$$
C q(\bar{R})= \begin{cases}1 \quad & \text { for } \quad \bar{R} \geq 1 \\ m_{0}+m_{1} \bar{R}+m_{2} \bar{R}^{2}+m_{3} \bar{R}^{3} & \text {, for } 0<\bar{R}<1\end{cases}
$$

where $\bar{R}$ is $\bar{R}_{b, 1}, \bar{R}_{e q}$ or $\bar{R}_{b, 2}$ (same in the following equations), $m_{0}, m_{1}, m_{2}$ and $m_{3}$ are respectively constants.

$\mathrm{Cy}(\bar{R})$ is generally expressed as (Zhang, 2018a and 2018b):

$$
\operatorname{Cy}(\bar{R})=\left\{\begin{array}{cl}
1 \quad, \quad \text { for } \quad & \bar{R} \geq 1 \\
a_{0}+\frac{a_{1}}{\bar{R}}+\frac{a_{2}}{\bar{R}^{2}} \quad, & \text { for } 0<\bar{R}<1
\end{array}\right.
$$

where $a_{0}, a_{1}$ and $a_{2}$ are respectively constants.

$S(\bar{R})$ is generally expressed as (Zhang, 2018a and 2018b):

$$
S(\bar{R})= \begin{cases}-1 \quad & \text { for } \quad \bar{R} \geq 1 \\ {\left[n_{0}+n_{1}\left(\bar{R}-n_{3}\right)^{n_{2}}\right]^{-1}} & , \text { for } n_{3}<\bar{R}<1\end{cases}
$$

where $n_{0}, n_{1}, n_{2}$ and $n_{3}$ are respectively constants.

For weak, medium-level and strong filtered liquid-pore wall interactions, the values of $R_{c r}$ were respectively taken as $3.5 \mathrm{~nm}, 10 \mathrm{~nm}$ and 20nm (Zhang, 2018a and 2018b). For different types of the filtered liquid-pore wall interaction, the values of the other parameters are respectively shown in Tables $1(\mathrm{a}-\mathrm{c})$.

Table 1(a) Liquid viscosity data for different liquid-pore wall interaction types (Zhang, 2018a and 2018b)

\begin{tabular}{|l|l|l|l|}
\hline Interaction & \multicolumn{1}{|c|}{$\mathrm{a}_{0}$} & \multicolumn{1}{c|}{$\mathrm{a}_{1}$} & $\mathrm{a}_{2}$ \\
\hline Strong & & & \\
\hline Medium & 1.8335 & -1.4252 & 0.5917 \\
\hline Weak & 1.0822 & -0.1758 & 0.0936 \\
\hline
\end{tabular}

Table 1(b) Liquid density data for different liquid-pore wall interaction types (Zhang, 2018a and 2018b)

\begin{tabular}{|l|c|c|c|c|}
\hline Interaction & $\mathrm{m}_{0}$ & $\mathrm{~m}_{1}$ & $\mathrm{~m}_{2}$ & $\mathrm{~m}_{3}$ \\
\hline Strong & & & & \\
\hline Medium & 1.43 & -1.723 & 2.641 & -1.347 \\
\hline Weak & 1.30 & -1.065 & 1.336 & -0.571 \\
\hline
\end{tabular}

Table 1(c) Liquid non-continuum property data for different liquidpore wall interaction types (Zhang, 2018a and 2018b)

\begin{tabular}{|l|l|c|c|c|}
\hline Interaction & \multicolumn{1}{c|}{$\mathrm{n}_{0}$} & $\mathrm{n}_{1}$ & $\mathrm{n}_{2}$ & $\mathrm{n}_{3}$ \\
\hline Strong & 0.4 & -1.374 & -0.534 & 0.035 \\
\hline Medium & -0.649 & -0.343 & -0.665 & 0.035 \\
\hline Weak & -0.1 & -0.892 & -0.084 & 0.1 \\
\hline
\end{tabular}

\section{RESULTS AND DISCUSSIONS}

\subsection{For liquid-particle separation}

The proposed membrane can be applied for liquid-particle separation for ultimate purification of liquids. In this case, the radius $R_{b, 1}$ of the branch pore is determined by the sizes of the filtered particles. As an example, all the results given in this section are for $\bar{R}_{b, 1}=0.3$.

Figure 3(a) plots the values of $\left(R_{b, 2} / R_{b, 1}\right)_{o p t}$ against $\lambda_{0}$ for different $\mathrm{N}$ for the weak liquid-pore wall interaction when $R_{r}=10 \mathrm{~nm}$. The value of $\left(R_{b, 2} / R_{b, 1}\right)_{\text {opt }}$ is significantly increased with the reduction of $\lambda_{0}$ and with the increase of $\mathrm{N}$; It can range between 10 and 30 , large enough for manufacturing tens of branch pores for each trunk pore.

Figure 3(b) plots the corresponding lowest dimensionless flow resistance $I_{f, \min }$ of the membrane for the weak liquid-pore wall interaction when $R_{r}=10 \mathrm{~nm}$ and the ratios $R_{b, 2} / R_{b, 1}$ are optimum. For a given $\lambda_{0}$, the value of $I_{f, \min }$ is significantly reduced with the increase of $\mathrm{N}$ especially when $\lambda_{0}$ is large. This shows the significant beneficial effect of the tree-type nanoporous filtering membrane with various branch pores in each pore tree as shown in Fig.1(a) in reducing the flow resistance and increasing the flux of the membrane. For a given $\mathrm{N}$, the value of $I_{f, \min }$ is significantly reduced with the reduction of $\lambda_{0}$. This shows the strong effect of the depth $l_{1}$ of the filtration pore on the flow resistance and thus on the flux of the present membrane.

Figure 4(a) shows the variations of the values of $\left(R_{b, 2} / R_{b, 1}\right)_{\text {opt }}$ with $\lambda_{0}$ similar as in Fig.3(a) for different $N$ for the medium liquid-pore 
wall interaction when $R_{r}=10 \mathrm{~nm}$. Figure 4(b) shows the variations of the corresponding lowest dimensionless flow resistance $I_{f, \min }$ of the membrane with $\lambda_{0}$ similar as in Fig.3(b) for the medium liquid-pore wall interaction when $R_{r}=10 \mathrm{~nm}$ and the ratios $R_{b, 2} / R_{b, 1}$ are optimum.

Figure 5(a) shows the variations of the values of $\left(R_{b, 2} / R_{b, 1}\right)_{o p t}$ with $\lambda_{0}$ similar as in Figs.3(a) and (b) for different $N$ for the strong liquid-pore wall interaction when $R_{r}=10 \mathrm{~nm}$. The comparisons among Fig.3(a), Fig.4(a) and Fig.5(a) show that for given operating conditions, a stronger liquid-pore wall interaction results in a lower value of $\left(R_{b, 2} / R_{b, 1}\right)_{\text {opt }}$.

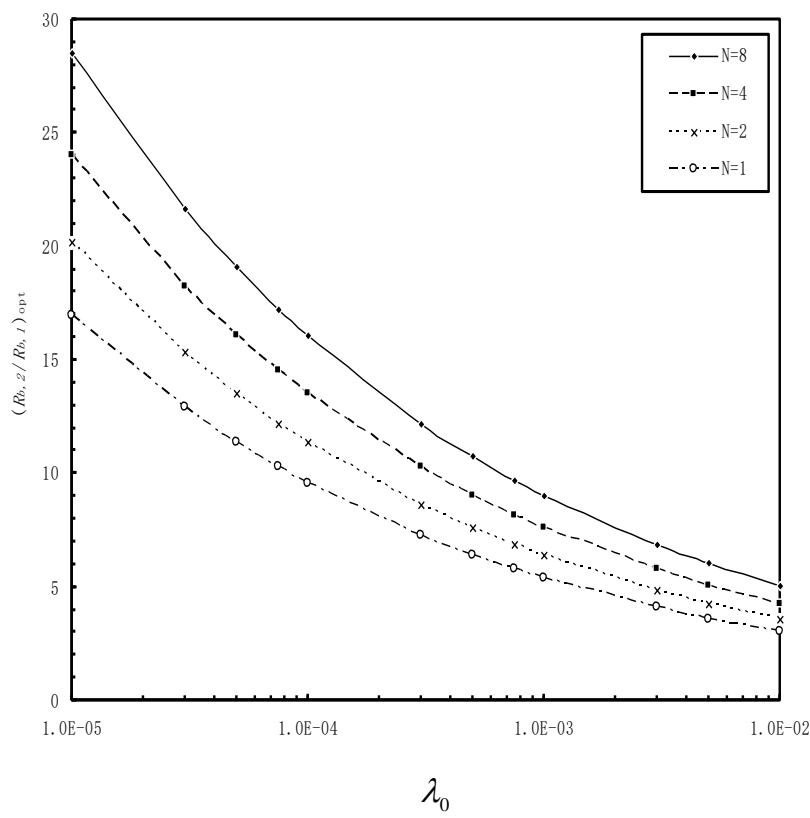

(a)

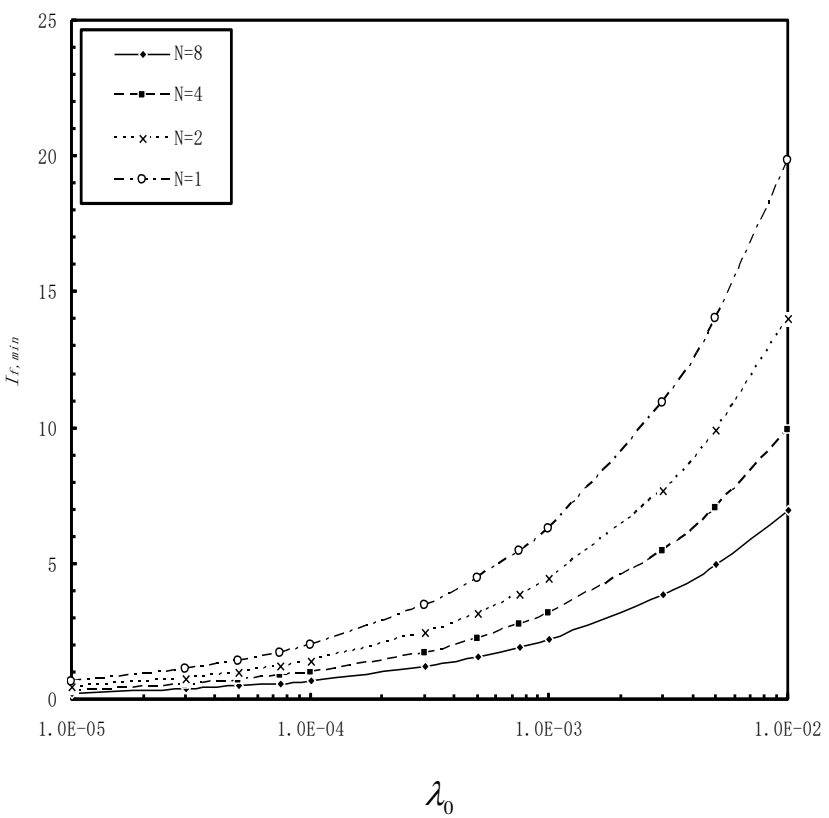

(b)

Fig. 3 Plots of the optimum values of $R_{b, 2} / R_{b, 1}$ and the corresponding lowest dimensionless flow resistance $I_{f, \min }$ of the membrane for the weak liquid-pore wall interaction when $R_{r}=10 \mathrm{~nm}$ and $\bar{R}_{b, 1}=0.3$.
Figure 5(b) shows the variations of the corresponding lowest dimensionless flow resistance $I_{f, \text { min }}$ of the membrane with $\lambda_{0}$ similar as in Figs.3(b) and 4(b) for the strong liquid-pore wall interaction when $R_{r}=10 \mathrm{~nm}$ and the ratios $R_{b, 2} / R_{b, 1}$ are optimum.

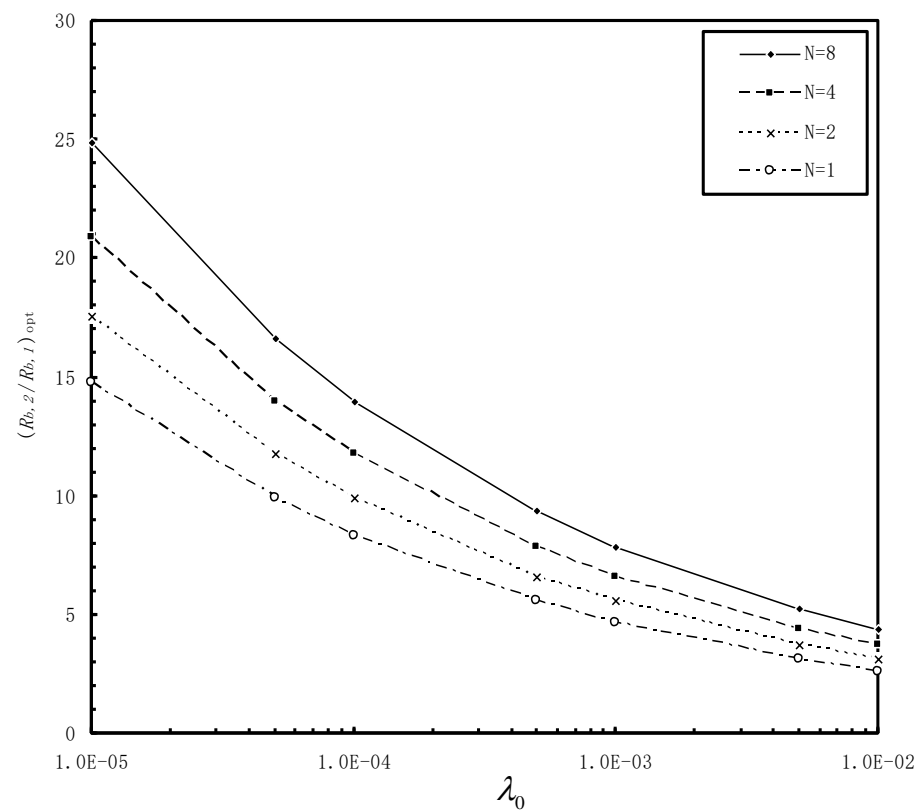

(a)

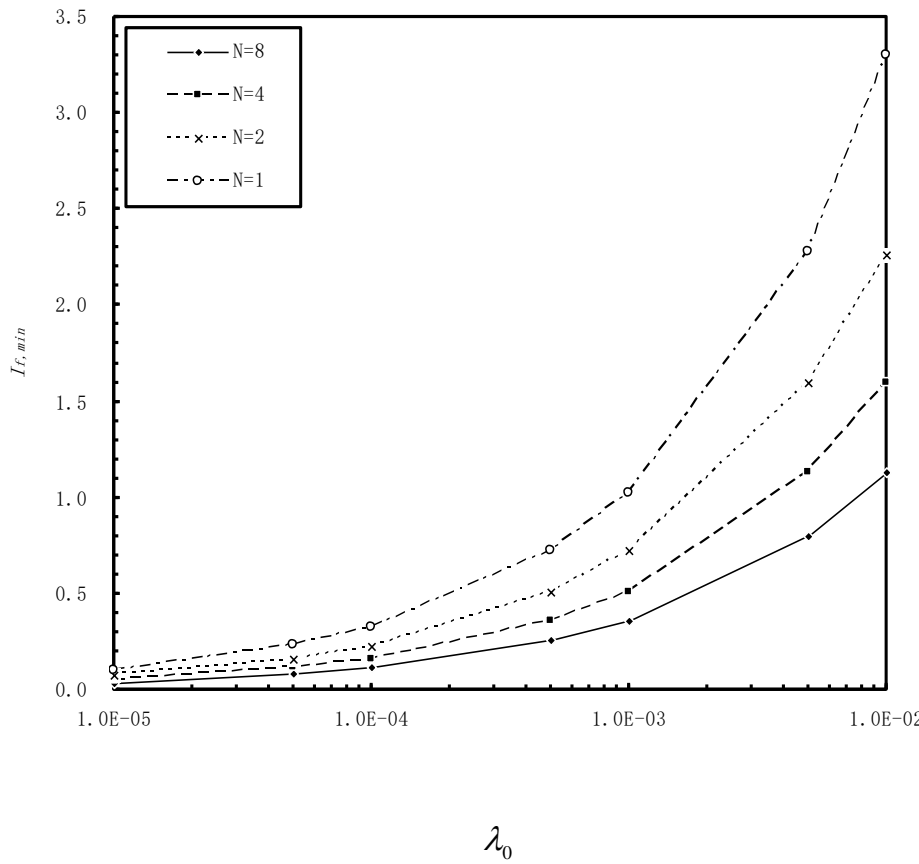

(b)

Fig. 4 Plots of the optimum values of $R_{b, 2} / R_{b, 1}$ and the corresponding lowest dimensionless flow resistance $I_{f, \min }$ of the membrane for the medium liquid-pore wall interaction when $R_{r}=10 \mathrm{~nm}$ and $\bar{R}_{b, 1}=0.3$.

Figure 6 shows the influences of the number $N$ of the branch pores in each pore tree on the lowest dimensionless flow resistance $I_{f, \min }$ of the present membrane for different liquid-pore wall interactions when $R_{r}=10 \mathrm{~nm}, \lambda_{0}=1 \times 10^{-3}$, and the ratios $R_{b, 2} / R_{b, 1}$ are optimum. It is shown that when $N$ is over large, the influence of $N$ on the flow resistance and thus on the flux of the membrane is weak for any liquidpore wall interaction; Otherwise, the increase of $N$ very significantly reduces the flow resistance and thus improves the flux of the membrane. 
For a weak liquid-pore wall interaction, $N$ can be 20 , for a medium liquidpore wall interaction, $N$ may be no more than 8 , while for a strong liquidpore wall interaction, $N$ may be no more than 5 .

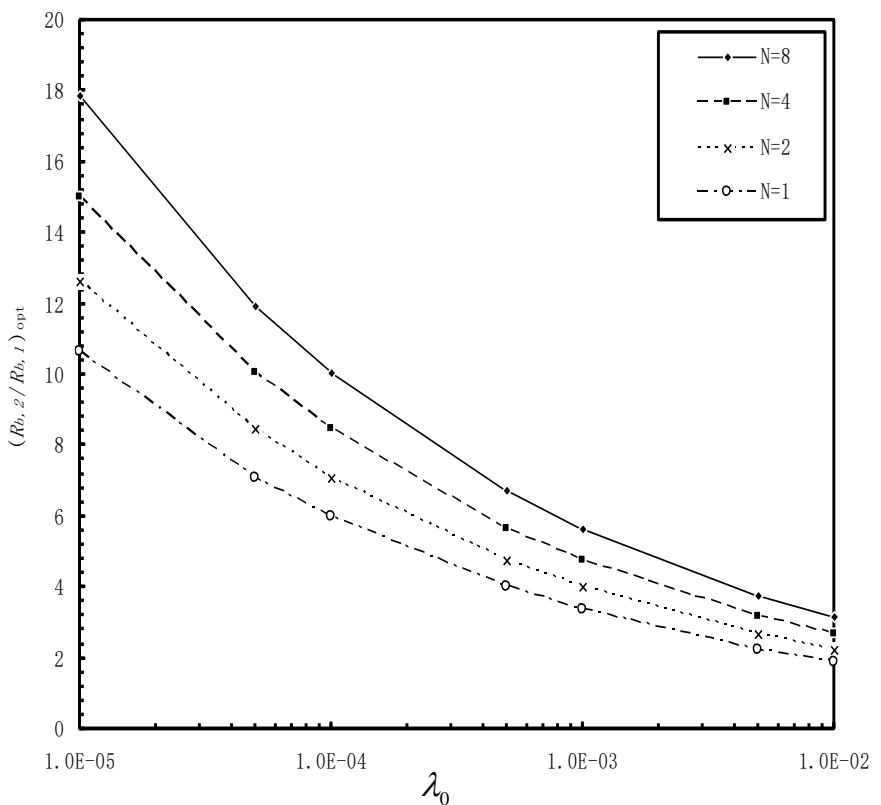

(a)

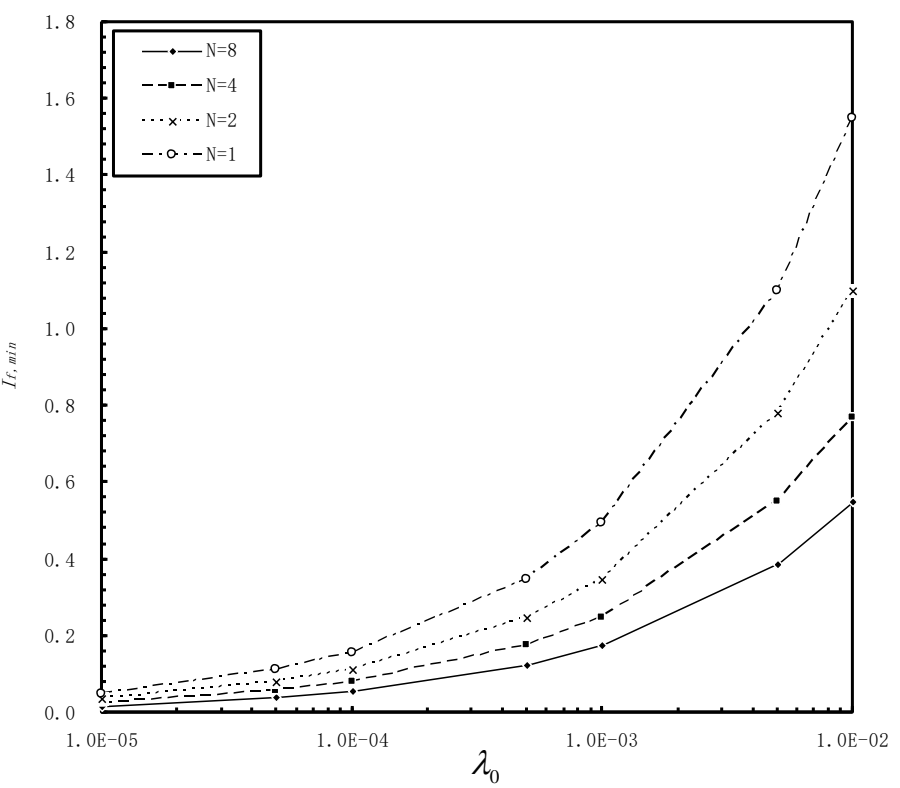

(b)

Fig. 5 Plots of the optimum values of $R_{b, 2} / R_{b, 1}$ and the corresponding lowest dimensionless flow resistance $I_{f, \min }$ of the membrane for the strong liquid-pore wall interaction when $R_{r}=10 \mathrm{~nm}$ and $\bar{R}_{b, 1}=0.3$.

\subsection{For liquid-liquid separation}

Similar with the membrane shown by Zhang (2017b), the present membrane might be applicable for a liquid-liquid separation. In this section, the results for the applicability of the membrane for liquid-liquid separations are presented.

Similar as shown by Zhang (2017b), for a liquid-liquid separation, it is here assumed that the mixed liquids have greatly different interactions with the pore wall in the membrane. The radius $R_{b, 2}$ of the trunk pore in the present membrane is determined as optimum according to the liquid with a weak interaction with the pore wall. In this circumstance, the liquid with a weak interaction with the pore wall may freely flow through the membrane, while the membrane might be highly resistive to the flow of the other liquids because of the much increased flow resistances. By this way, the mixed liquids should be well separated. In the flow through a nanochannel, there might be the effect of the interaction between the mixed liquids. In the present calculation, this effect is neglected. The flow resistances of the present membrane respectively for the liquids with weak, medium-level and strong interactions with the pore wall are here calculated to show the potential applications of the membrane for liquid-liquid separations.

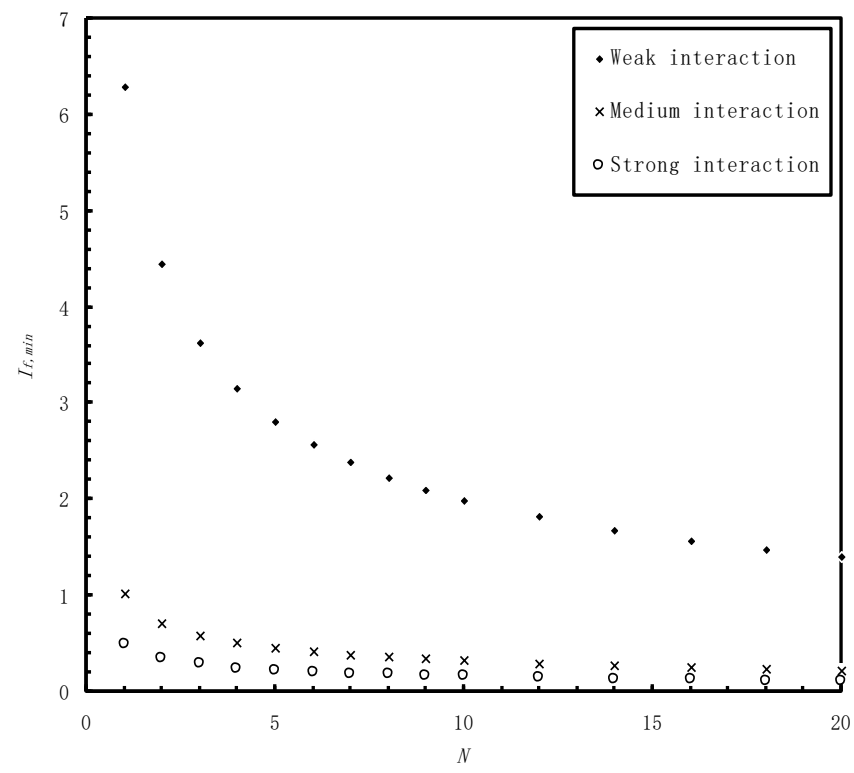

Fig. 6 Influences of the number $\mathrm{N}$ of the branch pores on the lowest dimensionless flow resistance $I_{f, \min }$ of the present membrane for different liquid-pore wall interactions when $R_{r}=10 \mathrm{~nm}, \bar{R}_{b, 1}=0.3$, $\lambda_{0}=1 \times 10^{-3}$, and the ratios $R_{b, 2} / R_{b, 1}$ are optimum.

For a liquid-liquid separation, the radius $R_{b, 2}$ of the trunk pore should be determined as follows:

$R_{b, 2}=R_{b, 1}\left(R_{b, 2} / R_{b, 1}\right)_{o p t}$

where $\left(R_{b, 2} / R_{b, 1}\right)_{o p t}$ is the optimum ratio of $R_{b, 2}$ to $R_{b, 1}$ for yielding the lowest flow resistance of the membrane for the liquid with a weak interaction with the pore wall and it is calculated as: $\left(R_{b, 2} / R_{b, 1}\right)_{\text {opt }}=$ $\left(R_{e q} / R_{b, 1}\right)\left(R_{b, 2} / R_{e q}\right)_{o p t}$. Here, $R_{e q}$ is solved from Eq.(1), and $\left(R_{b, 2} / R_{e q}\right)_{o p t}$ is calculated from Eq.(4). In all the calculations, it was taken that $R_{r}=10 \mathrm{~nm}$ and $\lambda_{0}=1 \times 10^{-3}$.

Figure 7 plots the optimum values of the radius $R_{b, 2}$ of the trunk pore determined according to the liquid with the weak interaction with the pore wall when $\lambda_{0}=1 \times 10^{-3}$. The optimum $R_{b, 2}$ value is nearly directly proportional to $R_{b, 1}$. For a given $R_{b, 1}$, it is significantly increased with the increase of $\mathrm{N}$.

Figures 8(a) and (b) show the variations of the dimensionless flow resistance $I_{f}$ of the membrane with the radius $R_{b, 1}$ of the branch pore respectively for the weak, medium and strong liquid-pore wall interactions i.e. for Liquid $\mathrm{A}$, Liquid $\mathrm{B}$ and Liquid $\mathrm{C}$ for $\mathrm{N}$ equal to 4 and 8 , when the radius $R_{b, 2}$ of the trunk pore is optimized according to the weak liquid-pore wall interaction i.e. Liquid $\mathrm{A}$ as shown in Fig.7. For any given $\mathrm{N}$, when $R_{b, 1}$ is below $2 \mathrm{~nm}$, the value of $I_{f}$ for Liquid $\mathrm{C}$ i.e. for the strong liquid-pore wall interaction is more than 100 or even more than 1000 times that for Liquid A i.e. for the weak liquid-pore wall interaction. This shows the potential application value of the present membrane for a liquid-liquid separation provided that the mixed liquids have largely different interactions with the pore wall. Greater $\mathrm{N}$ values result in significantly lower values of $I_{f}$ and then lower pressure drops 
and power losses on the membrane if the flux of the membrane is given in a liquid-liquid separation. This shows the benefit of the tree-type cylindrical-shaped nanoporous filtering membrane with multiple branch pores in each pore tree in a liquid-liquid separation. As shown by these figures, if the mixed liquids have less different interactions with the pore wall such as in separating Liquid $\mathrm{A}$ from Liquid $\mathrm{B}$, the required radius $R_{b, 1}$ of the branch pore should be much less e.g. no more than $0.5 \mathrm{~nm}$.

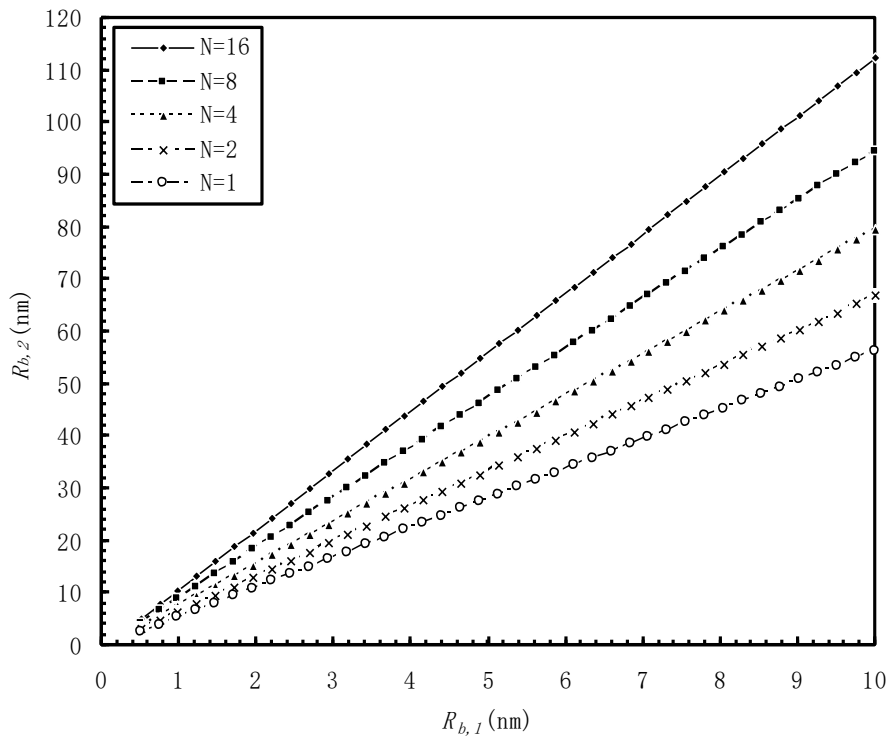

Fig. 7 Optimum $R_{b, 2}$ values determined according to the liquid with the weak interaction with the pore wall when $\lambda_{0}=1 \times 10^{-3}$.

Figures $8(a)$ and (b) show the variations of the dimensionless flow resistance $I_{f}$ of the membrane with the radius $R_{b, 1}$ of the branch pore respectively for the weak, medium and strong liquid-pore wall interactions i.e. for Liquid A, Liquid B and Liquid $\mathrm{C}$ for $\mathrm{N}$ equal to 4 and 8 , when the radius $R_{b, 2}$ of the trunk pore is optimized according to the weak liquid-pore wall interaction i.e. Liquid $\mathrm{A}$ as shown in Fig.7. For any given $\mathrm{N}$, when $R_{b, 1}$ is below $2 \mathrm{~nm}$, the value of $I_{f}$ for Liquid $\mathrm{C}$ i.e. for the strong liquid-pore wall interaction is more than 100 or even more than 1000 times that for Liquid A i.e. for the weak liquid-pore wall interaction. This shows the potential application value of the present membrane for a liquid-liquid separation provided that the mixed liquids have largely different interactions with the pore wall. Greater N values result in significantly lower values of $I_{f}$ and then lower pressure drops and power losses on the membrane if the flux of the membrane is given in a liquid-liquid separation. This shows the benefit of the tree-type cylindrical-shaped nanoporous filtering membrane with multiple branch pores in each pore tree in a liquid-liquid separation. As shown by these figures, if the mixed liquids have less different interactions with the pore wall such as in separating Liquid $\mathrm{A}$ from Liquid $\mathrm{B}$, the required radius $R_{b, 1}$ of the branch pore should be much less e.g. no more than $0.5 \mathrm{~nm}$.

\section{CONCLUSIONS}

An optimized tree-type cylindrical-shaped nanoporous filtering membrane is proposed. This membrane has two-leveled tree-structured pores across the thickness. The branch pores are parallel with its trunk pore, they are for filtration, and their number $\mathrm{N}$ can be relatively big depending on the requirement of the flux of the membrane. The trunk pore is for reducing the flow resistance of the membrane, and its radius is optimized for achieving the lowest flow resistance of the membrane.

An analysis is presented for the optimum ratio of the radius $R_{b, 2}$ of the trunk pore to the radius $R_{b, 1}$ of its branch pore and the corresponding lowest flow resistance $I_{f, \min }$ of the membrane. The equations for calculating the optimum $\left(l_{1}+l_{2}\right)$ and the corresponding value of $I_{f, \min }$ are derived. The calculations showed that the optimum value of the ratio $\left(l_{1}+l_{2}\right)$ can range between 10 and 30 , large enough for manufacturing tens of branch pores for each trunk pore. When the number $\mathrm{N}$ of the branch pores in each pore tree is not over large, the increase of $\mathrm{N}$ very significantly reduces the flow resistance and thus improves the flux of the membrane for any liquid-pore wall interaction; Otherwise, the influence of $\mathrm{N}$ on the flow resistance of the membrane is weak.

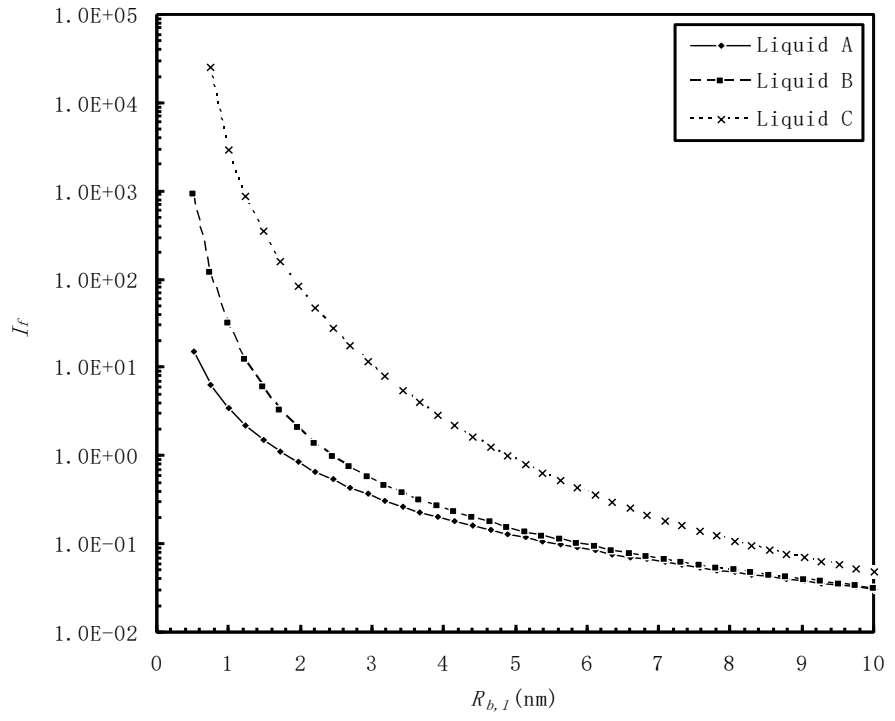

(a) $N=4$

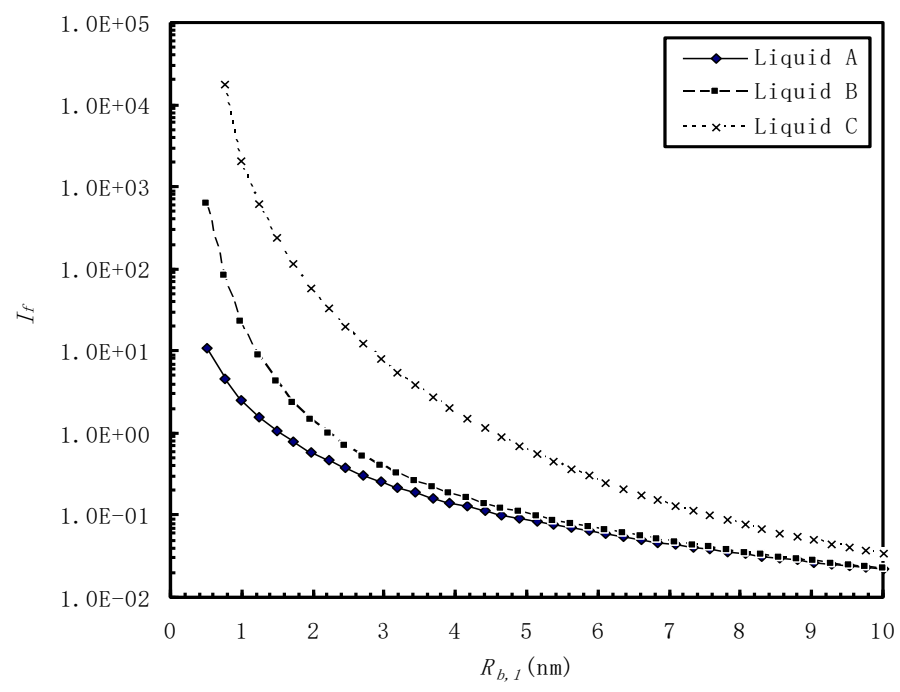

(b) $N=8$

Fig. 8 Plots of the dimensionless flow resistance of the membrane against the radius $R_{b, 1}$ of the branch pore respectively for the weak, medium and strong liquid-pore wall interactions i.e. for Liquid A, Liquid $\mathrm{B}$ and Liquid $\mathrm{C}$ for different $\mathrm{N}$ values.

The calculations also show that for any given number $\mathrm{N}$ of the branch pores in each pore tree, when the radius $R_{b, 1}$ of the branch pore is below $2 \mathrm{~nm}$, the flow resistance of the membrane for a weak liquidpore wall interaction can be more than 100 or even more than 1000 times that for a strong liquid-pore wall interaction. This shows the potential application value of the membrane for a liquid-liquid separation provided that the mixed liquids have largely different interactions with the pore wall. For separating the liquids with less different interactions with the 
pore wall, the required $R_{b, 1}$ value may be much less e.g. no more than $0.5 \mathrm{~nm}$. Greater $\mathrm{N}$ values bring more benefits in the performance of the membrane in a liquid-liquid separation such as lower pressure drops and power losses on the membrane for a given flux.

According to the analysis, for the same pressure drop $\Delta p$ on the membrane and the same membrane thickness $\left(l_{1}+l_{2}\right)$, the flux of the present optimized membrane should normally be much greater than that of the conventional membrane only with single cylindrical filtration pores. In other words, if both $\Delta p$ and the fluxes are the same, the thickness $\left(l_{1}+l_{2}\right)$ of the present optimized membrane should be significantly greater than that of the conventional membrane only with single cylindrical filtration pores; Thus, in this case, the mechanical strength of the present optimized membrane is considerably improved as compared with that of the conventional membrane. In actual application, we may need to find a balance between the mechanical strength and the flux of the present optimized membrane when determining the membrane thickness $\left(l_{1}+l_{2}\right)$.

Here, the obtained results and conclusions are for no liquid-pore wall interfacial slippage. However, the passing liquid might slip on the pore wall surface. For this case, a much more complicated analysis is required. In spite of this, the present results and conclusions should be of significant interest to the design and application of the mentioned membranes.

\section{REFERENCES}

Adiga, S. P., Jin, C. and Curtiss, L. A., Monteiro-Riviere, N. A., and Narayan, R. J., 2009, "Nanoporous Membranes for Medical and Biological Applications," Nanomedicine and Nanobiotechnology, 1, 568-581.

https://doi.org/10.1002/wnan.50

Baker, L. A., and Bird, S. P., 2008, "Nanopores: a Makeover for Membranes," Nature Nanotechnology, 3, 73-74.

https://doi.org/10.1038/nnano.2008.13

Biffinger, J. C., Ray, R., Little, B. and Ringeisen, B. R., 2007, "Diversifying Biological Fuel Cell Designs by Use of Nanoporous Filters," Environmental Science and Technology, 41, 1444-1449. https://doi.org/10.1021/es061634u

Fissel, W. H., Dubnisheva, A., Eldridge, A. N., Fleischman, A. J., Zydney, A. L., and Roy, S., 2009, "High-Performance Silicon Nanopore Hemofiltration Membranes," Journal of Membrane Science, 326, 58-63. https://doi.org/10.1016/j.memsci.2008.09.039

Jackson, E. A., and Hillmyer, M. A., 2010, "Nanoporous Membranes Derived from Block Copolymers: From Drug Delivery to Water Filtration," Acs Nano, 4, 3548-3553.

https://doi.org/10.1021/nn1014006

Li, N., Yu, S., Harrell, C., and Martin, C. R., 2004, "Conical Nanopore Membranes: Preparation and Transport Properties," Analytical Chemistry, 76, 2025-2030. https://doi.org/10.1021/ac035402e

Surwade, S. P., Smirnov, S. N., Vlassiouk, I. V., Unocic, R. R., Veith, G. M., Dai, S. and Mahurin, S. M., 2015, "Water Desalination Using Nanoporous Single-layer Graphene," Nature Nanotechnology, 10, 459464.

https://doi.org/10.1038/nnano.2015.37

Yang, S. Y., Ryu, I., Kim, H., Kim, J., Jang, S., and Russell, T., 2010, "Nanoporous Membranes with Ultrahigh Selectivity and Flux for the Filtration of Viruses," Advanced Materials, 18, 709-712. https://doi.org/10.1002/adma.200501500

Zhang, Y. B., 2016, "The Flow Equation for a Nanoscale Fluid Flow," International Journal of Heat and Mass Transfer, 92, 1004-1008. https://doi.org/10.1016/j.ijheatmasstransfer.2015.09.008

Zhang, Y. B., 2017a, "Transport in Nanotube Tree," International Journal of Heat and Mass Transfer, 114, 536-540. https://doi.org/10.1016/j.ijheatmasstransfer.2017.06.105

Zhang, Y. B., 2017b, "Influence of Pore Wall Surface Property on Flux of Nanoporous Filtering Membrane," Frontiers in Heat and Mass Transfer, 9, 26. http://dx.doi.org/10.5098/hmt.09.26

Zhang, Y. B., 2018a, "Optimum Design for Cylindrical-shaped nanoporous Filtration Membrane," International Communications in Heat and Mass Transfer, 96, 130-138. https://doi.org/10.1016/j.icheatmasstransfer.2018.06.003.

Zhang, Y. B., 2018b, "A Tree-type Cylindrical-shaped Nanoporous Filtering Membrane," Frontiers in Heat and Mass Transfer, 10, 16. http://dx.doi.org/10.5098/hmt.10.16 